\title{
Third generation gravitational-wave observatories and their science reach
}

\author{
Jim Hough • Harald Lueck • Michele Punturo • \\ B. S. Sathyaprakash
}

Published online: 14 December 2010

(C) Springer Science+Business Media, LLC 2010

Long-baseline interferometric gravitational-wave detectors have successfully reached their design goal in most of their frequency range. They have taken science quality data for several years achieving duty cycles of 70-95\%. Interesting science results from these instruments have appeared in many research papers and are reviewed by Fairhurst et al in this edition. The science runs have proved beyond doubt that not only one can reach the predicted sensitivity levels but also that these detectors can be operated pretty stably over long periods of time.

Indeed we are on the verge of direct observation of gravitational radiation from far out in the Universe and of opening the exciting new field of gravitational astronomy. In the coming decades, ultra-sensitive arrays of ground-based instruments and complementary space missions will make observations, discovering entirely unexpected phenomena, while providing new insight into many of the most profound astrophysical questions.

Acknowledging that so much progress has been made over the past decades, especially over the past three years in the context of the European Framework Programmefunded Einstein Telescope design study, and with strong encouragement from the Gravitational Wave International Committee (GWIC), we recognized that the time

\footnotetext{
J. Hough

University of Glasgow, Glasgow, Scotland, UK

H. Lueck

AEI, MPI, Hannover, Germany

M. Punturo

University of Perugia, Perugia, Italy

B. S. Sathyaprakash $(\varangle)$

Department of Physics and Astronomy, Cardiff University, 5, The Parade, Cardiff, CF24 3YB, UK

e-mail: B.Sathyaprakash@astro.cf.ac.uk
} 
was ripe to arrange for a special edition of the GRG Journal devoted to the astronomical and physical basis of the next generation of long-baseline gravitational wave detectors, those which will follow Advanced LIGO, Advanced Virgo, GEO600 and LCGT.

We invited chapters from colleagues working at the forefront of their research areas and these are presented in such a manner as to introduce the concepts to a non-expert reader.

This special edition begins with an overview of the why and how of third generation gravitational-wave observatories by Punturo and Lueck. Third generation observatories will have a very broad science reach. They should be able to detect a variety of different sources from supernovae to neutron star binaries and astronomical and primordial stochastic backgrounds. The edition continues with a series of articles on the science potential of third generation observatories and these discuss how an infrastructure like the Einstein Telescope could contribute to fundamental physics, cosmology and astrophysics. Of particular interest is a discussion of how the gravitational and electromagnetic windows are complementary to each other. Joint observation of a source with gravitational-wave interferometers, optical telescopes, radio antennas, neutrino observatories, gamma-ray and X-ray detectors, will immensely impact our understanding of cosmography, equation of state of exotic matter, cores of supernovae, etc.

The second part of the edition covers the technological advances required to build third generation detectors. Third generation observatories, such as the Einstein Telescope, should be based on infrastructure that allows the construction of multiple detectors but which is flexible enough for novel designs and configurations to be introduced over many decades. Achieving the sensitivity goal of the Einstein Telescope will require novel concepts in lasers, interferometry and thermal noise and mitigating gravity gradient noise in the $1-10 \mathrm{~Hz}$ band. Several ideas have been presented to address these overall issues as also a discussion of quantum non-demolition techniques and cryogenics.

The collection here is not exhaustive and many topics deserve further scrutiny. For example, a third generation detector will be an excellent tool for cosmography; use of non-classical light could be key to achieving the desired sensitivity. We hope that this special edition will trigger new research both on the science from and science of third generation observatories that will help us in better understanding the challenges to be met and rewards to reaped from building an observatory like the Einstein Telescope.

We are indebted to the authors, and many referees, who have worked very hard to bring this special edition to fruition. We would like to thank the GRG editors George Ellis and Herman Nicolai for their encouragement and Di Loureiro for her invaluable assistance with refereeing and production of the edition. 